\title{
A study of lymph nodes draining colorectal cancer using a two-stage inhibition of leucocyte migration technique
}

\author{
P. J. GUILlOU, T. G. BRENNAN, AND G. R. GILES \\ From the University Department of Surgery, St. James's Hospital, Leeds
}

\begin{abstract}
SUMMARY A two-stage technique for leucocyte migration inhibition has been developed which demonstrates the sensitization of lymph node lymphocytes to colorectal tumour extract. The studies have confirmed that some lymph nodes draining colorectal carcinomata contain sensitized lymphocytes. However, the degree of lymphocyte sensitization to tumour extract does not seem greater than that seen in lymph nodes of the small bowel mesentery or of the peripheral blood.
\end{abstract}

Lymph nodes draining malignant neoplasms may be a focus for host resistance against the tumour (Crile, 1968; Deodhar, Crile, and Esseltyn, 1972). Specific cellular immunity to tumour antigens may readily be demonstrated in nodes draining experimental animal tumours (Humphrey, Barker, Bohesch, Fetter, Anderson, and Boehm, 1971) but there have been few reports on the presence of antitumour immunity in lymph nodes draining human malignant neoplasms, despite the possible therapeutic implications of such a finding. The test of leucocyte migration inhibition represent one technique for the demonstration of specific cellular immunity to antigens (Rosenberg and David, 1970), and has previously been employed by us for the demonstration of cellular hypersensitivity of the peripheral blood lymphocytes of tumour-bearing patients (Guillou and Giles, 1973). However, unlike peripheral leucocytes, suspensions of cells from lymph nodes do not contain a significant migrating cell population (Thor and Dray, 1968) and cannot be used directly in a one-stage test. We have therefore developed a two-stage test for the demonstration of migratory inhibitory factor (MIF) release from specifically sensitized lymphocytes present in lymph nodes draining colorectal tumours.

\section{Materials and Methods}

CLINICAL GROUPS

Leucocyte migration studies designed to investigate the sensitization of both peripheral and lymph node lymphocytes to tumour-associated antigens were

Received for publication 18 December 1974. performed in 14 patients suffering from adenocarcinoma of the colon and rectum. The results of these experiments were compared with the results of identical investigations on the peripheral blood and lymph node lymphocytes of 10 patients suffering from benign gastrointestinal disease who served as control subjects. The mean age of the patients with colorectal carcinoma was 65 years (range 39 years to 70 years), and that of the control subjects was 56 years (range 40 years to 75 years).

The patients with carcinoma were staged according to the extent of the disease using the classification devised by Dukes (1957). One patient was classified as stage $A$, seven patients as stage $B$, five patients as stage $C$, and the remaining patient with hepatic metastases was stage $D$. The site of the primary lesion was the rectum in four patients, the sigmoid colon in five patients, the transverse colon in three patients, and in the remaining two patients the tumour was in the caecum. The group of control subjects was comprised of patients undergoing surgery for peptic ulceration, cholelithiasis, or diverticular disease.

For all patients under investigation, the peripheral blood studies were performed on the morning of operation. At laparotomy an attempt was made to obtain lymph nodes from the root of the mesentery and from a paracolonic site in the control subjects and also from the mesentery and a group of nodes directly draining the tumour in the patients with colorectal carcinoma. Attempts to obtain nodes from each site in both groups of patients were not uniformly successful but at least one node was obtained from each patient; 14 nodes were obtained from the control subjects and 25 nodes from the patients with colorectal cancer. 
SINGLE-STAGE TECHNIQUE OF LEUCOCYTE MIGRATION INHIBITION FOR PERIPHERAL

CIRCULATING LEUCOCYTES

The method used in these studies for the investigation of peripheral lymphocyte sensitization to tumour antigens was a modification of the technique of Bendixen and Soberg (1969). Peripheral blood leucocytes were separated from $20 \mathrm{ml}$ of defibrinated venous blood by sedimentation with one half this volume of dextran 150 in $0.9 \%$ saline (Dextraven 150, Fisons Ltd). The leucocytes were then washed at least four times in TC 199 prepared at a $\mathrm{pH}$ of 7.4 without the addition of antibiotics. This leucocyte suspension was adjusted to a cell concentration of $10^{7}$ per $\mathrm{ml}$ and drawn into $10 \mu \mathrm{l}$ capillary pipettes (Drummond Microcaps, Drummond Scientific Co, USA) which were then heat-sealed at one end. Following centrifugation at $100 \mathrm{~g}$ for five minutes the capillaries were cut at the cell-fluid interface. The leucocyte-containing portions were placed in the wells of microtissue culture plates (Sterilin Ltd) with the open end of the tube facing centrally. After each well had been filled with approximately $0.5 \mathrm{ml}$ of medium, it was covered with a sterile cover slip and sealed with silicone grease. All cultures were incubated for 20 hours at $37^{\circ} \mathrm{C}$.

For each subject studied, capillaries were prepared in quadruplicate for incubation in each of the following media: (a) $10 \%$ homologous $\mathrm{AB}$ serum in TC 199; (b) 10\% homologous AB serum in TC 199 with the addition of normal colonic mucosal extract; (c) $10 \%$ homologous $\mathrm{AB}$ serum in TC 199 with the addition of tumour extract; (d) $10 \%$ autologous serum in TC 199; (e) $10 \%$ autologous serum in TC 199 with the addition of normal colonic mucosal extract; (f) $10 \%$ autologous serum in TC 199 with the addition of tumour extract.

\section{TWO-STAGE TECHNIQUE OF LEUCOCYTE MIGRATION INHIBITION FOR LYMPH-NODE LYMPHOCYTES}

Suspensions of ce!ls extracted from lymph nodes do not contain a migrating cell population and so cannot be used directly in the one-stage technique as described above for peripheral lymphocytes. Nevertheless, if sensitized lymphocytes are present in a cell population then they will release migratory inhibitory factor irrespective of the nature of the vessel containing them. Subsequently the supernatant from such a culture may be used as the medium for the culture of a non-immune migrating cell population and the presence of migratory inhibitory factor detected by any resultant inhibition of migration.

In these experiments (fig 1) each lymph node was macerated under sterile conditions and a cell suspension obtained by filtration through a sterile $30 \mu$ diameter sieve. The suspension was corrected to a cell density of $2 \times 10^{6}$ per $\mathrm{ml}$ and separate $0.5 \mathrm{ml}$ aliquots were placed into each of six tissue

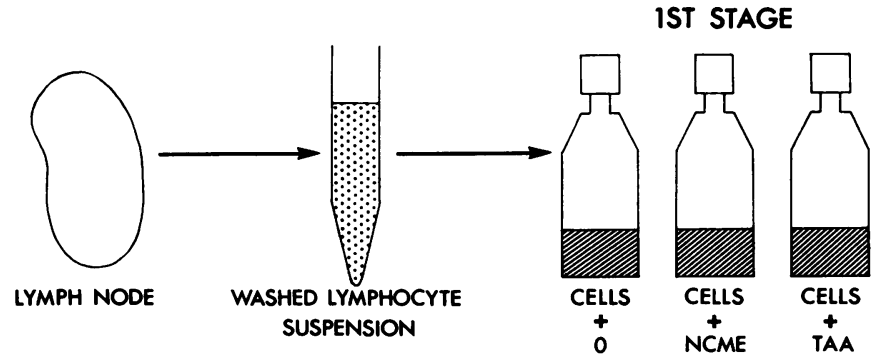

INCUBATE 72 HOURS

NORMAL PERIPHERAL BLOOD LEUCOCYTES CAPILLARY TUBE

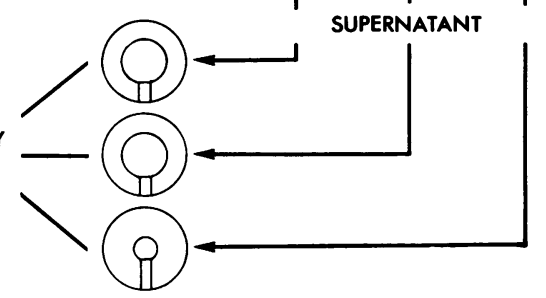

2ND STAGE
Fig 1 The basic technique of two-stage leucocyte migration test for lymph node lymphocytes. 
culture flasks (Falcon Plastics Ltd). Of pooled homologous $\mathrm{AB}$ serum $0.5 \mathrm{ml}$ was added to each of three of the flasks and $0.5 \mathrm{ml}$ of autologous serum added to each of the remaining three. If insufficient cells were available to perform all six cultures then experiments with autologous serum were omitted. Antigens were then added to the appropriate flasks such that cell cultures had now been prepared in each of the media described under the single-stage test. These cultures were incubated at $37^{\circ} \mathrm{C}$ for 72 hours, after which the supernatant was removed and corrected to a pH of 7.4 if necessary. The supernatants were placed in separate microtissue culture wells containing capillaries packed with the peripheral leucocytes obtained in the manner previously described from one of a group of several healthy control subjects who had consistently shown no inhibition of migration in response to either of the antigens in the single-stage test. Each supernatant was again cultured with quadruplicate capillaries at $37^{\circ} \mathrm{C}$ for 20 hours as for the one-stage test.

During the 20-hour incubation period leucocytes migrated from the open ends of the capillary tubes and these areas were measured by planimetry following projection onto paper at a constant magnification. The mean area of migration for each group of four capillaries was calculated and the results were expressed as a migratory index which is the mean area of migration in the presence of tissue extract divided by the mean area of migration obtained in the absence of tissue extract, ie, migratory index $=\frac{M x}{M o}$. In all these experiments homologous AB serum was obtained from a pool of eight healthy donors. Tissue extracts were added to the media at a final concentration in culture of $50 \mu \mathrm{g}$ of protein per $\mathrm{ml}$. This concentration was a non-toxic dose which did not influence migration of the leucocytes from a group of healthy individuals during preliminary dose-response experiments reported elsewhere (Guillou and Giles, 1973).

\section{PREPARATION OF TISSUE EXTRACTS}

Tissue extracts were prepared from pooled samples of colorectal adenocarcinomata and pooled samples of normal colonic mucosa removed from the same operative specimens as the tumour samples but from a site at least $10 \mathrm{~cm}$ away from the tumour in each case. Antigen isolation was performed by the perchloric acid extraction technique of Freed and Taylor (1972). Antisera were then raised in rabbits to each of the tissue extracts and the presence of tumour-associated antigens demonstrated by the technique of double diffusion on Ouchterlony plates. The protein concentration of each extract was estimated by the method of Lowry, Rosebrough,
Farr, and Randall (1951). Immunodiffusion procedures were performed on each sample of antigen from each pool of tumour obtained.

\section{Results}

In this investigation the indices of migration obtained for the control subjects have been used to establish the normal range of migration inhibition on exposure to the two types of tissue extracts. Thus the $95 \%$ confidence limits (mean \pm 2 standard deviations) were calculated for the experiments with each antigen and serum. The migration indices obtained for the patients were then examined with particular reference to those which fell below the lower 2.5 percentile for the range of results obtained for the control group. Those results falling below this limit were taken to indicate a positive result to the tissue extract.

\section{PERIPHERAL BLOOD STUDIES}

Culture in homologous $A B$ serum

The mean migratory index for control subjects in the

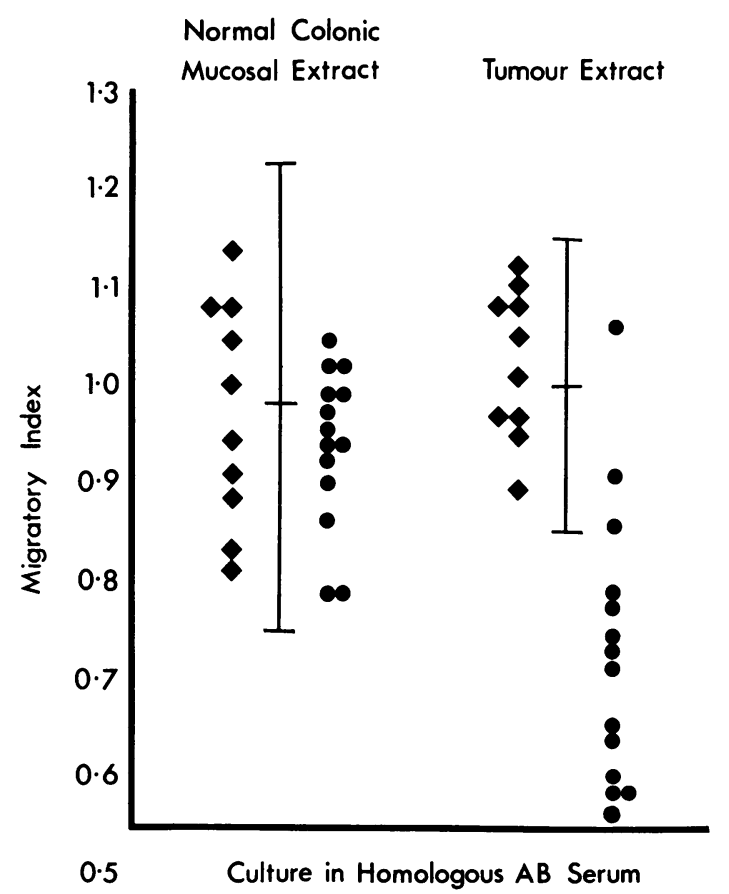

Fig 2 The migratory indices of lymphocytes extracted from the peripheral blood after exposure to normal colonic mucosal extract and tumour extract and cultured in homologous AB serum. (Control subjects: closed diamonds; patients with cancer: closed circles.) 
presence of normal colonic mucosal extract was $0.98 \pm 0.23(2 \times$ standard deviation $)$. Using these values as the normal range it can be seen that none of the patients with cancer had migratory indices below the lowest limit (fig 2).

The mean migratory index in the presence of the tumour extract for the control subjects was $1.00 \pm$ $0 \cdot 15$ (fig 2). Eleven of the 14 patients with cancer had migratory indices below this lower limit of the normal range and thus exhibit sensitization to the tumour extract.

\section{Culture in autologous serum}

The mean migratory index of the control subjects on exposure to the normal colonic mucosal extract was $0.97 \pm 0.13$. Two of the patients with cancer had migratory indices below the lower limit of normality (fig 3) and both these patients had shown sensitization to tumour extract when cultured in homologous AB serum.

On exposure to tumour extract the mean migra-

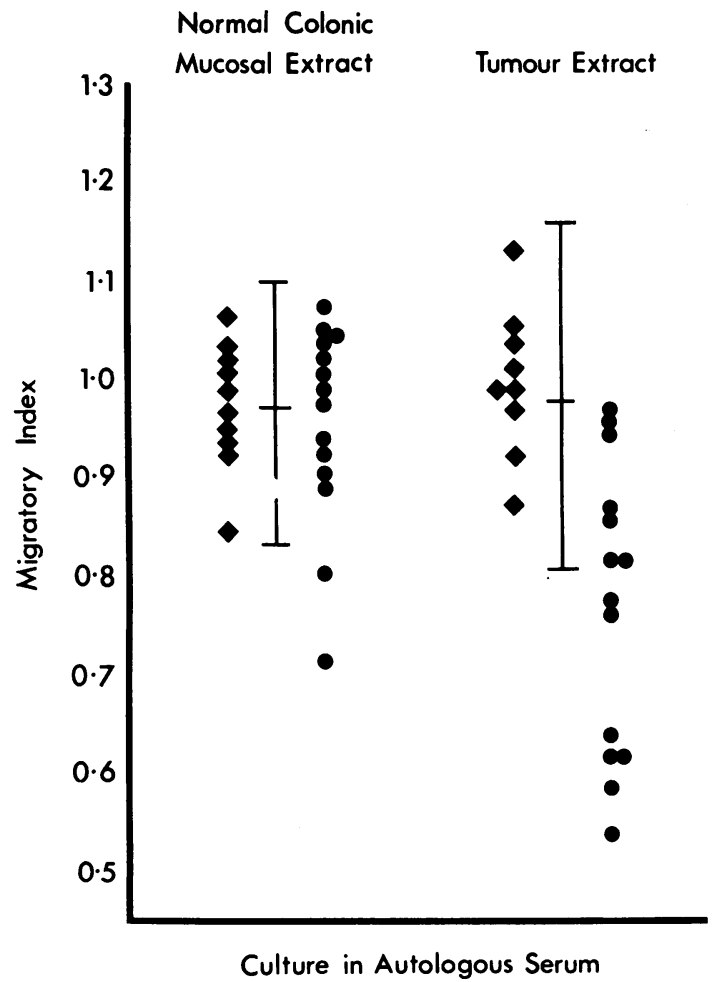

Fig 3 The migratory indices of lymphocytes extracted from peripheral blood after exposure to normal colonic mucosal extract and tumour extract and cultured in autologous serum. (Control subjects: closed diamonds; patients with cancer: closed circles.) tory index in control subjects was $0.98 \pm 0.17$ and seven of the patients with cancer had values below the level of this range and all of these had shown sensitization to the tumour extract when cultured in homologous $\mathrm{AB}$ serum.

\section{LYMPH NODE STUDIES}

\section{Culture in homologous $A B$ serum}

Using the two-stage test the mean migratory index for the 14 lymph nodes obtained from the control group was $1.0 \pm 0.2$ on exposure to the normal colonic mucosal extract and $0.97 \pm 0.18$ when cultured with tumour extract.

Six of the lymph nodes from the patients with cancer had migratory indices below the limits found in the control subjects on exposure to normal colonic mucosal extract (fig 4). However, 18 of the 25 nodes from the patients with cancer had indices below the limits for the control group on exposure to tumour extract. All six nodes which had

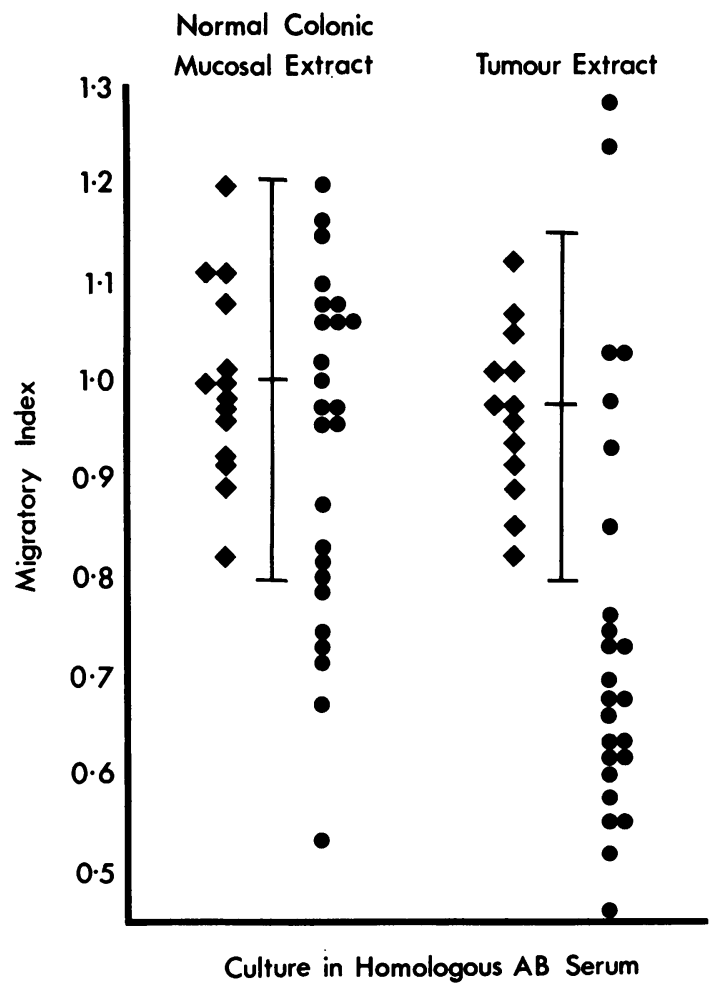

Fig 4 The migratory indices of lymph node lymphocytes using the two-stage technique and culturing with normal colonic mucosal extract or tumour extract in homologous $A B$ serum. (Control subjects: closed diamonds; patients with cancer: closed circles.) 
shown a positive response to normal colonic mucosal extract also demonstrated marked inhibition of migration in response to the tumour extract (fig 4).

\section{Culture in autologous serum}

Seven of the control lymph nodes yielded a sufficient number of cells for studies to be performed in autologous serum as well as in pooled homologous $\mathrm{AB}$ serum. The mean migratory index given by the control nodes in autologous serum in the presence of normal colonic mucosal extract was $1 \cdot 01 \pm 0 \cdot 25$. Of the 25 nodes from the patients with colorectal cancer, the cells of only 15 nodes could be studied in both autologous serum and pooled homologous AB serum. None of these nodes gave migratory indices outside the $95 \%$ confidence limits for the control group when cultured in autologous serum in the presence of normal colonic mucosal extract (fig 5).

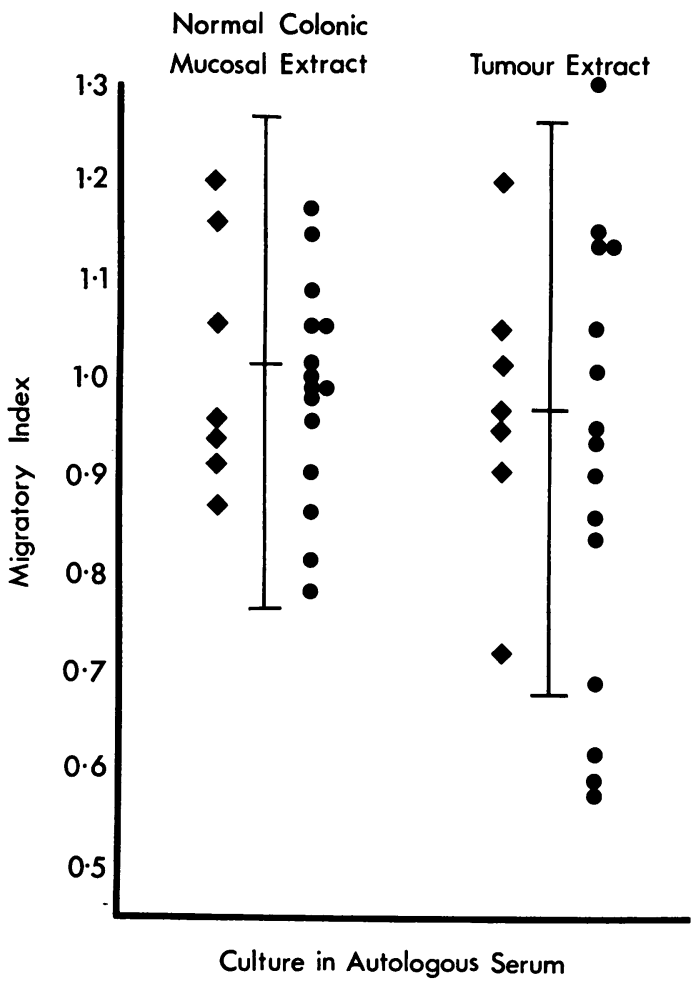

Fig 5 The migratory indices of lymph node lymphocytes using the two-stage technique and culturing with normal colonic mucosal extract or tumour extract in autologous serum. (Control subjects: closed diamonds; patients with cancer: closed circles.)
On culture in the presence of tumour extract the mean migratory index for the control group was $0 \cdot 97 \pm 0 \cdot 29$. Only three of the 15 nodes from patients with colorectal cancer demonstrated migratory indices less than the $95 \%$ confidence limits for the control group (fig 5).

Further analysis of the results obtained for the 15 nodes which were examined with regard to their response to tumour extract both in homologous and in autologous serum reveals that 12 of the nodes showed marked inhibition of migration when cultured in homologous AB serum whereas only four such nodes exhibited a positive response in autologous serum. This difference was statistically significant at the $5 \%$ level using Fisher's test $(n=$ $30, d=15$ ). Furthermore none of the three nodes which failed to show sensitization in homologous $A B$ serum expressed such immunity in autologous serum.

INHIBITION OF MIGRATION PRODUCED IN RELATION TO THE SITE OF ORIGIN OF THE LYMPHOCYTES

In all lymph node studies, experimental conditions such as cell concentration, concentration of tissue antigens, and leucocyte donors in the two-stage test were identical. Thus it may be permissible to compare the migratory indices obtained for lymph nodes from the root of the mesentery and paracolonic sites in the same patient. These comparisons have been applied only to the results obtained from culture in homologous AB serum. There were five control subjects and 11 patients with cancer where a complete set of results was obtained for peripheral blood, mesenteric nodes, and paracolonic lymph nodes. These results are detailed in table I. Leucocyte migration in the supernatant from control cells cultured with both normal colonic mucosal extract and tumour extract showed minor variations but there were no significant differences between peripheral blood, mesenteric, and paracolonic lymph nodes (table I). Of the 11 patients with cancer, two showed sensitization to normal colonic mucosal extract in the mesenteric and paracolonic nodes (patients 6 and 9, table I). Eight of these 11 peripheral blood samples contained lymphocytes sensitized to tumour extract (mean migration index = $0.72 \pm 0.15$ ). In the same manner only two of the mesenteric nodal lymphocyte samples failed to inhibit migration with tumour extract (mean migration index $=0.69 \pm 0 \cdot 17$ ). However, four of the 11 paracolonic nodes did not inhibit migration (mean migration index $=0.83 \pm 0.25$ ).

Five patients had tumours apparently confined to the bowel wall (Duke's B), five patients had nodal metastases (Duke's C), and one patient had dis- 


\begin{tabular}{|c|c|c|c|c|c|c|c|}
\hline \multirow[t]{2}{*}{ Subjects } & \multirow[t]{2}{*}{ Duke's Stage } & \multicolumn{2}{|l|}{ Peripheral Blood } & \multicolumn{2}{|l|}{ Mesenteric Nodes } & \multicolumn{2}{|l|}{ Paracolonic Nodes } \\
\hline & & $\begin{array}{l}\text { Normal Colonic } \\
\text { Mucosal Extract }\end{array}$ & $\begin{array}{l}\text { Pooled Tumour } \\
\text { Extract }\end{array}$ & $\begin{array}{l}\text { Normal Colonic } \\
\text { Mucosal Extract }\end{array}$ & $\begin{array}{l}\text { Pooled Tumour } \\
\text { Extract }\end{array}$ & $\begin{array}{l}\text { Normal Colonic } \\
\text { Mucosal Extract }\end{array}$ & $\begin{array}{l}\text { Pooled Tumour } \\
\text { Extract }\end{array}$ \\
\hline \multirow[t]{2}{*}{$\begin{array}{l}\text { Controls } \\
1 \text { J.R. } \\
2 \text { N.W. } \\
3 \text { F.H. } \\
4 \text { W.F. } \\
5 \text { E.R. }\end{array}$} & $\begin{array}{l}- \\
- \\
-\end{array}$ & $\begin{array}{l}1.08 \\
0.82 \\
1.08 \\
1.08 \\
0.84\end{array}$ & $\begin{array}{l}1.09 \\
0.96 \\
1.00 \\
1.1 \\
0.89\end{array}$ & $\begin{array}{l}0.91 \\
0.92 \\
0.82 \\
1.08 \\
0.98\end{array}$ & $\begin{array}{l}0.93 \\
1.07 \\
0.97 \\
0.91 \\
1.01\end{array}$ & $\begin{array}{l}0.97 \\
1.2 \\
1.11 \\
0.89 \\
0.99\end{array}$ & $\begin{array}{l}0.89 \\
1.05 \\
0.97 \\
1.01 \\
0.97\end{array}$ \\
\hline & Mean \pm SD & $0.98 \pm 0.13$ & $1.00 \pm 0.08$ & $0.94 \pm 0.90$ & $0.97 \pm 0.06$ & $1.03 \pm 0.12$ & $0.97 \pm 0.05$ \\
\hline \multicolumn{8}{|c|}{ Patients with Colorectal Cancer } \\
\hline \multirow[t]{2}{*}{$\begin{array}{l}1 \text { L.S. } \\
2 \text { M.L. } \\
3 \text { G.R. } \\
4 \text { C.S. } \\
5 \text { H.S. } \\
6 \text { O.B. } \\
7 \text { E.S. } \\
8 \text { B.H. } \\
9 \text { R.S. } \\
10 \text { V.R. } \\
11 \text { E.O. }\end{array}$} & $\begin{array}{l}\text { C } \\
\text { B } \\
\text { C } \\
\text { B } \\
\text { B } \\
\text { C } \\
\text { C } \\
\text { B } \\
\text { B } \\
\text { D } \\
\text { C }\end{array}$ & $\begin{array}{l}0.99 \\
0.97 \\
0.86 \\
0.93 \\
0.98 \\
1.02 \\
0.95 \\
0.79 \\
0.95 \\
1.04 \\
0.79\end{array}$ & $\begin{array}{l}0.75 \\
0.65 \\
0.73 \\
0.79 \\
0.71 \\
0.59 \\
0.86 \\
0.78 \\
0.51 \\
1.06 \\
0.59\end{array}$ & $\begin{array}{l}0.86 \\
0.96 \\
0.82 \\
0.98 \\
1.00 \\
0.69 \\
1.06 \\
1.15 \\
0.53 \\
1.16 \\
0.98\end{array}$ & $\begin{array}{l}0.62 \\
0.74 \\
0.52 \\
0.73 \\
0.46 \\
0.55 \\
0.93 \\
0.67 \\
0.64 \\
1.03 \\
0.7\end{array}$ & $\begin{array}{l}1.08 \\
0.96 \\
1.10 \\
1.06 \\
1.08 \\
0.73 \\
0.74 \\
0.72 \\
0.78 \\
1.20 \\
1.02\end{array}$ & $\begin{array}{l}0.69 \\
0.76 \\
1.03 \\
0.98 \\
0.68 \\
0.62 \\
0.64 \\
0.56 \\
0.73 \\
1.29 \\
1.23\end{array}$ \\
\hline & Mean \pm SD & $0.9 \pm 0.09$ & $0.72 \pm 0.15$ & $0.92 \pm 0.19$ & $0.69 \pm 0.17$ & $0.95 \pm 0.17$ & $0.83 \pm 0.25$ \\
\hline
\end{tabular}

Table Migration indices of leucocytes in $10 \%$ homologous $A B$ serum

seminated disease (Duke's D). Of the patients with 'B stage' disease, all five had mesenteric nodal leucocytes which showed sensitization to tumour extract compared with four of the paracolonic nodal cultures (table $\mathrm{I})$. In the patients with ' $\mathrm{C}$ stage' disease, four blood samples, four mesenteric and three paracolonic nodes contained lymphocytes apparently sensitized to tumour extract. The patient with disseminated disease failed to show inhibition of migration using peripheral blood, mesenteric, or paracolonic nodes.

\section{Discussion}

The role of lymphoid tissue and lymph nodes in the humoral and cellular defences against bacterial infection is well known. Lymph nodes act primarily as a filter of tumour cells, delaying the extension of the neoplasm beyond a particular region. However, it is clear that the poor prognosis of patients with cancer and involved lymph nodes refutes the idea that lymph nodes are efficient in their filtration function. This has led to the concept that there is a therapeutic advantage offered by prophylactic node dissection which is based on the assumption that not only are lymph nodes an ineffective barrier to tumour cells, but they may also act as a source of further dissemination. This concept has been the guiding principle in the development of surgical techniques for the treatment of most types of cancer for over 50 years (Halsted, 1895; Handley, 1922).

It now appears that the therapeutic advantages of this approach with regard to cure or survival after surgery for a particular cancer are questionable. Indeed during the last two decades there has been growing scepticism as to the validity of this type of surgical approach, particularly with regard to such commonly occurring neoplasms as mammary cancer (Crile, 1968). Obviously, it can be speculated that tumour cells bypass lymph nodes and proceed to more distant nodes or pass into the bloodstream to distant organs. Equally it is becoming clear that factors other than the apparent extent of the cancer must influence the prognosis. These factors include biological activity of the tumour itself as demonstrated by the variable histological grading. Similarly, it has been suggested that changes within the draining lymph nodes themselves may have some relevance to the ultimate outcome. Black, Opler, and Speer (1956) have regarded the histological demonstration of sinus histiocytosis within a draining lymph node as an excellent prognostic sign regardless of the presence of nodal metastases. The significance of these histological criteria is much debated and refuted by other studies (Berg, 1956) but the question does arise as to whether lymph nodes draining tumours have some function other than acting as a barrier and whether the lymphocytes residing in these nodes have an adverse effect on tumour emboli. There is considerable evidence that some types of lymphocytes are cytotoxic to neoplastic cells in vitro (Hellström, Hellström, Evans, Heppner, Pierce, and Young, 1969) and suggestive evidence that this may also occur in vivo (Deodhar et al, 1972). Lymphocyte function in lymph nodes draining tumours of man has not been investigated in depth though it does not 
seem that the cells residing in these nodes are in a high degree of immunological excitation (Guillou, Brennan, and Giles, 1973).

However, the experimental work reported by Alexander, Bensted, Delorme, Hall, and Hodgett (1969) would suggest that lymphocytes residing in lymph nodes draining tumours are specially sensitized to the antigens of the associated tumour. Lymphocytes removed from stimulated nodes and labelled with ${ }^{51} \mathrm{Cr}$ will home into the regional nodes of syngeneic animals immunized with the same antigen (Hall and Morris, 1963; Delorme, Hodgett, Hall, and Alexander, 1969; Zatz and Lance, 1971). Thus it would appear that lymphocytes should demonstrate evidence of antigenic sensitization even though their immunocompetence remains in doubt.

It is known that sensitized lymphocytes are capable of releasing soluble lymphocyte mediators (migratory inhibiting factor) on exposure to antigen (Pick and Turk, 1972). The test of migratory inhibition has previously been applied to the demonstration of tumour sensitization of peripheral lymphocytes in a variety of tumours (Cochran, Spilg, Mackie, and Thomas, 1973; Andersen, Bjerrum, Bendixen, Schiødt, and Dissing, 1970) and, in particular, to colorectal cancer (Guillou and Giles, 1973). However, technical problems arise when the test is applied to the cell population of lymph nodes, for the resident population of migratory cells appears to be too small. In this study the migratory inhibition test was modified into two stages. In the first stage the lymphocytes were reacted with tumour antigen in cell culture to permit the release of migratory inhibitory factor into the supernatant. This supernatant was then used in microculture with migratory cells from a normal individual who was not sensitized to the tumour antigen. The migratory inhibitory factor acted upon these cells and in some cases was present in sufficiently high concentration to inhibit migration.

The studies on the peripheral blood lymphocytes produced results which were similar to those described in a previous report from this laboratory (Guillou and Giles, 1973). In patients with cancer, sensitization to normal colonic mucosal extract was not seen, whereas a majority of peripheral lymphocyte cultures produced inhibition of migration with tumour extract. The 'blocking effect' of autologous serum was shown in four of these patients who failed to show migration inhibition when compared with studies in homologous $\mathrm{AB}$ serum. This blocking effect by the autologous serum from patients with cancer on cellular hypersensitivity reactions in vitro has now achieved widespread recognition (Hellström et al, 1969; Whittaker, Rees, and Clark, 1971). The nature of the blocking factor is not entirely clear but has been suggested to consist of circulating antigen, antibody, or antigen-antibody complexes.

The results obtained for the culture of lymphocytes from lymph nodes are somewhat more difficult to interpret. Nodal lymphocytes from six patients were apparently sensitized to normal colonic mucosal extract. It seems likely that this results from contamination of the normal colonic mucosal extract by tumour extract since both extracts are usually obtained from the same specimen. Though $72 \%$ of nodal specimens would produce migration inhibition to tumour extract in homologous $\mathrm{AB}$ serum, only $20 \%$ showed sensitization when cultured with autologous serum. While this blocking effect of autologous serum from patients with cancer is exhibited against a test system in vitro, it does suggest that sensitized lymphocytes in vivo either in the peripheral blood or in the nodes may not be able to exert their fullest immunological function.

Despite the clear-cut experimental evidence of specific nodal sensitization for tumour, the data from patients with colorectal cancer are less convincing. In studies in 11 patients where a complete examination of blood and mesenteric and paracolonic nodes was obtained, lymph node lymphocytes failed to show especially marked inhibition of migration. In fact, fewer paracolonic nodes showed sensitization to tumour extract even though they were directly draining the tumour.

The findings would suggest that sensitization of local nodes may occur early in the progression of malignant disease, as evidenced by the observation of sensitization of paracolonic nodes in four out of five stage B cases studied. However, this lymphocyte sensitization may be lost when the lymph nodes become involved with tumour metastases. One possible explanation for this phenomenon is that the agent which inhibits the expression of cellmediated antitumour immunity is the presence of tumour cells themselves, though humoral factors such as antigen-antibody complexes cannot be excluded.

The results of this investigation do not support the concept that lymphocytes residing in lymph nodes directly draining a tumour are more sensitized than lymphocytes from other sites. In all but two patients the degree of migration inhibition brought about by paracolonic node lymphocytes was less than that resulting from culture of the same number of mesenteric node lymphocytes from the same patient. Whilst this is clearly a semiquantitative analysis, it would seem that the paracolonic node cells are unlikely to be capable of an efficient local antitumour response. It is suggested that there can be no justification on immunological grounds for abandoning lymph node resection in the manage- 


\section{ment of colorectal cancer.}

\section{References}

Alexander, P., Bensted, J., Delorme, E. J., Hall, J. G., and Hodgett, J. (1969). The cellular immune response to primary sarcoma in rats. II. Abnormal responses of nodes draining the tumour. Proc. roy. Soc. B., 174, 237-251.

Andersen, V., Bjerrum, O., Bendixen, G., Schivdt, T., and Dissing, I. (1970). Effect of autologous mammary tumour extracts on human leukocyte migration in vitro. Int. J. Cancer, 5, 357-363.

Bendixen, G., and Soberg, M. (1969). A leucocyte migration technique for in vitro detection of cellular (delayed type) hypersensitivity in man. Dan. med. Bull., 16, 1-6.

Berg, J. W. (1956). Sinus histiocytosis: a fallacious measure of host resistance to cancer. Cancer (Philad.), 9, 935-939.

Black, M. M., Opler, S. R., and Speer, F. D. (1956). Structural representations of tumor-host relationships in gastric carcinoma. Surg. Gynec. Obstet., 102, 599-603.

Cochran, A. J., Spilg, W. G. S., Mackie, R. M., and Thomas, C. E. (1972). Postoperative depression of tumour directed cellmediated immunity in patients with malignant disease. Brit. med. J., 4, 67-70.

Crile, G., Jr. (1968). Results of simple mastectomy without irradiation in the treatment of operative stage $I$ cancer of the breast. Ann. Surg., 168, 330-336.

Delorme, E. J., Hodgett, J., Hall, J. G., and Alexander, P. (1969). The cellular immune response to primary sarcoma in rats. I. The significance of large basophilic cells in the thoracic duct lymph following antigenic challenge. Proc. roy. Soc. B., 174, 229-236.

Deodhar, S. D., Crile, G., Jr., and Esseltyn, C. B., Jr. (1972). Study of the tumour cell-lymphocyte interaction in patients with breast cancer. Cancer (Philad.), 29, 1321-1325.

Dukes, C. E. (1957). Discussion on major surgery in carcinoma of the rectum with or without colostomy, excluding the anal canal and including the rectosigmoid. Proc. roy. Soc. Med., 50, 1031-1035.

Freed, D. L. J., and Taylor, G. (1972). Carcinoembryonic antigen in faeces. Brit. med. J., 1, 85-87.
Guillou, P. J., Brennan, T. G., and Giles, G. R. (1973). Phytohaemagglutinin-stimulated transformation of peripheral and lymphnode lymphocytes in patients with gastrointestinal cancer. Brit. J. Surg., 60, 745-749.

Guillou, P. J., and Giles, G. R. (1973). Inhibition of leucocyte migration by tumour-associated antigens of the colon and rectum. Gut, 14, 733-738.

Hall, J. G., and Morris, B. (1963). The lymph-borne cells of the immune response. Quart. J. exp. Physiol., 48, 235-247.

Halsted, W. S. (1907). The results of radical operations for the cure of carcinoma of breast. Ann. Surg., 46, 1-19.

Handley, W. S. (1922). Cancer of the Breast and its Treatment. Hoeber, New York. Murray, London.

Hellström, I., Hellström, K. E., Evans, C. A., Heppner, G. M., Pierce, G. H., and Young, J. P. S. (1969). Serum mediated protection of neoplastic cells from inhibition of lymphocytes immune to their tumor specific antigens. Proc. nat. Acad. Sci. (Wash.), 62, 362-368.

Humphrey, L. J., Barker, C., Bohesch, C., Fetter, D., Amerson, J. R., and Boehm, O. R. (1971) Immunologic competence of regional lymph nodes in patients with mammary cancer. Ann. Surg., 174, 383-391.

Lowry, O. H., Rosebrough, N. J., Farr, A. L., and Randall, R. J. (1951). Protein measurement with the folin-phenol reagent. J. biol. Chem., 193, 265-275.

Pick, E., and Turk, J. L. (1972). The biological activities of soluble lymphocyte products. Clin. exp. Immunol., 10, 1-23.

Rosenberg, S. A., and David, J. R. (1970). Inhibition of leucocyte migration: an evaluation of this in vitro assay of delayed hypersensitivity in man to a soluble antigen. J. Immunol., 105, 1447-1452.

Thor, D. E., and Dray, S. (1968). The cell migration inhibition correlate of delayed hypersensitivity: conversion of human nonsensitive lymph node cells to sensitive cells with an RNA extract. J. Immunol., 101, 469-480.

Whittaker, M. G., Rees, K., and Clark, C. G. (1971). Reduced lymphocyte transformation in breast cancer. Lancet, 1, 892-893.

Zatz, M. M., and Lance, E. M. (1971). The distribution of 51Crlabeled lymphocytes into antigen stimulated mice.J. exp. Med., 134, 224-241. 